\title{
On the Critical Coupling Strength for Kuramoto Oscillators
}

\author{
Florian Dörfler and Francesco Bullo
}

\begin{abstract}
The celebrated Kuramoto model captures various synchronization phenomena in biological and man-made dynamical systems of coupled oscillators. It is well-known that there exists a critical coupling strength among the oscillators at which a phase transition from incoherency to synchronization occurs. This paper features three contributions. First, we characterize and distinguish the different notions of synchronization used throughout the literature and formally introduce the concept of phase cohesiveness as an analysis tool and performance index for synchronization. Second, we review the vast literature providing necessary, sufficient, implicit, and explicit estimates of the critical coupling strength in the finite and infinitedimensional case. Finally, we present the first explicit necessary and sufficient condition on the critical coupling strength to achieve synchronization in the finite-dimensional Kuramoto model for an arbitrary distribution of the natural frequencies. The multiplicative gap in the synchronization condition yields a practical stability result determining the admissible initial and the guaranteed ultimate phase cohesiveness as well as the guaranteed asymptotic magnitude of the order parameter.
\end{abstract}

\section{The Kuramoto Model of Coupled Oscillators}

A classic model for the synchronization of coupled oscillators is due to Kuramoto [1]. The Kuramoto model considers $n \geq 2$ coupled oscillators each represented by a phase variable $\theta_{i} \in \mathbb{T}^{1}$, the 1 -tours, and a natural frequency $\omega_{i} \in \mathbb{R}$. The system of coupled oscillators obeys the dynamics

$$
\dot{\theta}_{i}=\omega_{i}-\frac{K}{n} \sum_{j=1}^{n} \sin \left(\theta_{i}-\theta_{j}\right), \quad i \in\{1, \ldots, n\},
$$

where $K>0$ is the coupling strength among the oscillators.

The Kuramoto model (1) finds application in various biological synchronization phenomena, and we refer the reader to the excellent reviews [2], [3] for various references. Recent technological applications of the Kuramoto model include motion coordination of particles [4], synchronization in coupled Josephson junctions [5], deep brain stimulation [6], and transient stability analysis of power networks [7].

Kuramoto himself analyzed the model (1) based on the order parameter $r e^{\mathrm{i} \psi} \triangleq \frac{1}{n} \sum_{j=1}^{n} e^{\mathrm{i} \theta_{j}}$, which corresponds the centroid of all oscillators when represented as points on the unit circle in $\mathbb{C}^{1}$. The magnitude $r$ of the order parameter can be understood as a measure of synchronization: if all oscillators are perfectly synchronized with identical angles $\theta_{i}(t)$, then $r=1$, and if all oscillators are spaced equally on the unit circle, then $r=0$. With the order parameter, the Kuramoto model (1) can be written in the insightful form

$$
\dot{\theta}_{i}=\omega_{i}-K r \sin \left(\theta_{i}-\psi\right), \quad i \in\{1, \ldots, n\} .
$$

This work was supported in part by NSF grants IIS-0904501 and CNS0834446.

Florian Dörfler and Francesco Bullo are with the Center for Control, Dynamical Systems and Computation, University of California at Santa Barbara, Santa Barbara, CA 93106, \{dorfler, bullo\}eengineering.ucsb.edu
Equation (2) gives the intuition that the oscillators synchronize by coupling to a mean field represented by the order parameter $r e^{\mathrm{i} \psi}$. Intuitively, for small coupling strength $K$ each oscillator rotates with its natural frequency $\omega_{i}$, whereas for large coupling strength $K$ all angles $\theta_{i}(t)$ will be entrained by the mean field $r e^{\mathrm{i} \psi}$. This phase transition from incoherency to synchronization occurs for some critical coupling $K_{\text {critical }}$ and has been the source of numerous articles starting with Kuramoto's own insightful analysis [1], [8]. For instance, since $r \leq 1$, no solution of (2) of the form $\dot{\theta}_{i}(t)=\dot{\theta}_{j}(t)$ can exist if $K<\left|\omega_{i}-\omega_{j}\right| / 2$. Hence, $K \geq\left|\omega_{i}-\omega_{j}\right| / 2$ is a necessary synchronization condition and a lower bound for $K_{\text {critical }}$. Various necessary and sufficient bounds on $K_{\text {critical }}$ for both the on-set and the ultimate stage of synchronization have been derived in the vast literature.

The contributions of this paper are three-fold.

First, we characterize, distinguish, and relate different concepts of synchronization and their analysis methods, which are studied and employed in the networked control, physics, and dynamical systems communities. In particular, we review the concepts of phase synchronization and frequency synchronization, and introduce the notion of phase cohesiveness. In essence, a solution to the Kuramoto model (1) is phase cohesive if all angles are bounded within a (possibly rotating) arc of fixed length. The notion of phase cohesiveness provides a powerful analysis tool for synchronization and can be understood as a performance index for synchronization similar to the order parameter.

As second contribution, we review the extensive literature on the Kuramoto model, and present various necessary, sufficient, implicit, and explicit estimates of the critical coupling strength for the finite and infinite-dimensional Kuramoto model in a unified language [1]-[4], [7]-[25]. Aside from the comparison of the different estimates of the critical coupling strength, the second purpose of this literature review is the comparison of the different analysis techniques.

As third, final, and main contribution of this paper, we provide an explicit necessary and sufficient condition on the critical coupling strength to achieve exponential synchronization in the finite-dimensional Kuramoto model for an arbitrary distribution of the natural frequencies $\omega_{i}$. In particular, synchronization occurs for $K>K_{\text {critical }}=$ $\omega_{\max }-\omega_{\min }$, where $\omega_{\max }$ and $\omega_{\min }$ are the maximum and minimum natural frequency, respectively. The multiplicative gap $K_{\text {critical }} / K$ determines the admissible initial and the guaranteed ultimate level of phase cohesiveness as well as the guaranteed asymptotic magnitude $r$ of the order parameter. In particular, the ultimate level of phase cohesiveness can be made arbitrary small by increasing the multiplicative gap $K_{\text {critical }} / K$. This result resembles the control-theoretic concept of practical stability if $K$ and $K_{\text {critical }}$ are understood 
as a synchronization-enhancing gain and as a measure for the desynchronizing non-uniformity among the oscillators. Furthermore, our main result includes estimates on the exponential synchronization rate for phase and frequency synchronization as well as a local stability property.

Compared to the authors' earlier work in power networks [7], the third contribution proves necessity of the sufficient bound on the critical coupling derived in [7] and doubles the estimate for the region of attraction presented in [7].

The remainder of this paper is organized as follows. Section II reviews different concepts of synchronization and provides a motivating example. Section III reviews the literature on the critical coupling strength in the Kuramoto model, and Section IV presents our main result. The simulation studies in Section V compare our bound with other explicit and necessary or implicit and exact bounds known in the literature. Finally, Section VI concludes the paper.

Notation: The torus is the set $\left.\left.\mathbb{T}^{1}=\right]-\pi,+\pi\right]$, where $-\pi$ and $+\pi$ are associated with each other, an angle is a point $\theta \in \mathbb{T}^{1}$, and an arc is a connected subset of $\mathbb{T}^{1}$. The product set $\mathbb{T}^{n}$ is the $n$-dimensional torus. With slight abuse of notation, let $\left|\theta_{1}-\theta_{2}\right|$ denote the geodesic distance between two angles $\theta_{1} \in \mathbb{T}^{1}$ and $\theta_{2} \in \mathbb{T}^{1}$. For $\gamma \in[0, \pi]$, let $\Delta(\gamma) \subset \mathbb{T}^{n}$ be the set of angle arrays $\left(\theta_{1}, \ldots, \theta_{n}\right)$ with the property that there exists an arc of length $\gamma$ containing all $\theta_{1}, \ldots, \theta_{n}$ in its interior. Thus, an angle array $\theta \in \Delta(\gamma)$ satisfies $\max _{i, j \in\{1, \ldots, n\}}\left|\theta_{i}-\theta_{j}\right|<\gamma$. For $\gamma \in[0, \pi]$, we also define $\bar{\Delta}(\gamma)$ to be the union of the phase-synchronized set $\left\{\theta \in \mathbb{T}^{n} \mid \theta_{i}=\theta_{j}, i, j \in\{1, \ldots, n\}\right\}$ and the closure of the open set $\Delta(\gamma)$. Hence, $\theta \in \bar{\Delta}(\gamma)$ satisfies $\max _{i, j \in\{1, \ldots, n\}}\left|\theta_{i}-\theta_{j}\right| \leq \gamma$; the case $\theta \in \bar{\Delta}(0)$ corresponds simply to $\theta$ taking value in the phase-synchronized set. Finally, given an $n$-tuple $\left(x_{1}, \ldots, x_{n}\right)$, let $x_{\max }$ and $x_{\min }$ be the corresponding maximum and minimum elements.

\section{SynCHRONIZATION NOTIONS AND CONCEPTS}

Different levels of synchronization are typically distinguished for the Kuramoto model (1). The case when all angles $\theta_{i}(t)$ converge exponentially to a common angle $\theta_{\infty} \in \mathbb{T}^{1}$ as $t \rightarrow \infty$ is referred to as exponential phase synchronization and can only occur if all natural frequencies are identical. If the natural frequencies are non-identical, then each pairwise distance $\left|\theta_{i}(t)-\theta_{j}(t)\right|$ can converge to a constant value, but this value is not necessarily zero. The following concept of phase cohesiveness addresses exactly this point. A solution $\theta: \mathbb{R}_{\geq 0} \rightarrow \mathbb{T}^{n}$ to the Kuramoto model (1) is phase cohesive if there exists a length $\gamma \in[0, \pi[$ such that $\theta(t) \in \bar{\Delta}(\gamma)$ for all $t \geq 0$, i.e., at each time $t$ there exists an arc of length $\gamma$ containing all angles $\theta_{i}(t)$. A solution $\theta: \mathbb{R}_{\geq 0} \rightarrow \mathbb{T}^{n}$ achieves exponential frequency synchronization if all frequencies $\dot{\theta}_{i}(t)$ converge exponentially fast to a common frequency $\dot{\theta}_{\infty} \in \mathbb{R}$ as $t \rightarrow \infty$. Finally, a solution $\theta: \mathbb{R}_{\geq 0} \rightarrow \mathbb{T}^{n}$ achieves exponential synchronization if it is phase cohesive and it achieves exponential frequency synchronization.

If a solution $\theta(t)$ achieves exponential frequency synchronization, all phases asymptotically become constant in a rotating coordinate frame with frequency $\dot{\theta}_{\infty}$, or equivalently, all phase distances $\left|\theta_{i}(t)-\theta_{j}(t)\right|$ asymptotically become constant. Hence, the terminology phase locking is sometimes also used in the literature to define a solution $\theta: \mathbb{R}_{>0} \rightarrow \mathbb{T}^{n}$ that satisfies $\dot{\theta}_{i}(t)=\dot{\theta}_{\infty}[11],[15],[20]$ or $\theta_{i}(t)-\theta_{j}(t)=$ constant for all $i, j \in\{1, \ldots, n\}$ and for all $t \geq 0$ [9], [19], [21], [23], [24]. Other commonly used terms in the vast synchronization literature include full, exact, or perfect synchronization (or even phase locking [3]) for phase synchronization and frequency locking, frequency entrainment, or partial synchronization for frequency synchronization.

In the networked control community, boundedness of angular distances and consensus arguments are typically combined to prove frequency synchronization [7], [12], [13], [15], [16], [18]. Our analysis in Section IV makes this approach to synchronization explicit by distinguishing between phase cohesiveness and frequency synchronization. Note that phase synchronization is simply the extreme case of phase cohesiveness with $\lim _{t \rightarrow \infty} \theta(t) \in \bar{\Delta}(0)$, and phase cohesiveness can also be understood as a performance measure for synchronization. Indeed, if the magnitude $r$ of the order parameter is understood as an average performance index for synchronization, then phase cohesiveness can be understood as a worst-case performance index. Since the order parameter $r e^{\mathrm{i} \psi}$ is the centroid of all phasors $e^{\mathrm{i} \theta_{j}} \in \mathbb{C}^{1}$, it is contained in the convex hull of the arc of length $\gamma$, as illustrated in Figure 1. Hence, the magnitude of the order parameter can be easily related to a guaranteed level of phase cohesiveness.

Lemma II.1 Consider an array of angles $\theta=\left(\theta_{1}, \ldots, \theta_{n}\right) \in$ $\mathbb{T}^{n}, n \geq 2$, and compute the magnitude of the order parameter $r(\theta)=\frac{1}{n}\left|\sum_{j=1}^{n} e^{\mathrm{i} \theta_{j}}\right|$. The following statements hold:

1) if $\theta \in \bar{\Delta}(\gamma)$ for some $\gamma \in[0, \pi[$, then $r(\theta) \in$ $[\cos (\gamma / 2), 1]$; and conversely

2) if $r(\theta) \in[0,1]$ and $\theta \in \bar{\Delta}(\pi)$, then $\theta \in \bar{\Delta}(\gamma)$ for some $\gamma \in[2 \arccos (r(\theta)), \pi]$.

In the physics and dynamical systems community exponential synchronization or phase locking is usually analyzed in relative coordinates. For instance, since the average frequency $\frac{1}{n} \sum_{i=1}^{n} \dot{\theta}_{i}(t)=\frac{1}{n} \sum_{i=1}^{n} \omega_{i} \triangleq \omega_{\text {avg }}$ is constant, the Kuramoto model (1) is sometimes [19], [20] analyzed with respect to a rotating frame in the coordinates $\xi_{i}=\theta_{i}-\omega_{\mathrm{avg}} t$ $(\bmod 2 \pi), i \in\{1, \ldots, n\}$, corresponding to a deviation from the average angle. The existence of an exponentially stable one-dimensional (due to translational invariance) equilibrium manifold in $\xi$-coordinates then implies local stability of phase-locked solutions and exponential synchronization. Alternatively, the translational invariance can be removed by formulating the Kuramoto model (1) in grounded coordinates

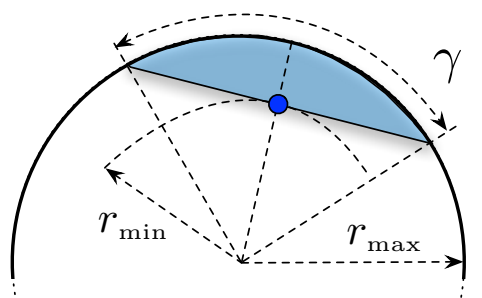

Fig. 1. Schematic illustration of an arc of length $\gamma \in[0, \pi]$, its convex hull (shaded), and a location $\bullet$ of the corresponding order parameter $r e^{\mathrm{i} \psi}$. 
$\delta_{i}=\theta_{i}-\theta_{n}$, for $i \in\{1, \ldots, n-1\}$ [7], [21]. We refer the reader to [7, Lemma IV.1] for a geometrically rigorous characterization of the grounded $\delta$-coordinates and the relation of exponential stability in $\delta$-coordinates and exponential synchronization in $\theta$-coordinates.

The following example of two oscillators illustrates the notion of phase cohesiveness, applies graphical synchronization analysis techniques, and points out various important geometric subtleties occurring on the compact state space $\mathbb{T}^{2}$.

Example II.1 (Two oscillators) Consider $n=2$ oscillators with $\omega_{2}>\omega_{1}$. We restrict our attention to angles contained in an open half-circle: for angles $\theta_{1}, \theta_{2}$ with $\left|\theta_{2}-\theta_{1}\right|<\pi$, we define the angular difference $\theta_{2}-\theta_{1}$ to be the number in ] $-\pi, \pi$ [ with magnitude equal to the geodesic distance $\left|\theta_{2}-\theta_{1}\right|$ and with positive sign iff the counter-clockwise path length from $\theta_{1}$ to $\theta_{2}$ on $\mathbb{T}^{1}$ is smaller than the clockwise path length. With this definition the two-dimensional Kuramoto dynamics $\left(\dot{\theta}_{1}, \dot{\theta}_{2}\right)$ can be reduced to the scalar difference dynamics $\dot{\theta}_{2}-\dot{\theta}_{1}$. After scaling time as $t \mapsto t\left(\omega_{2}-\omega_{1}\right)$ and introducing $\kappa=K /\left(\omega_{2}-\omega_{1}\right)$ the difference dynamics are

$$
\frac{d}{d t}\left(\theta_{2}-\theta_{1}\right)=f\left(\theta_{2}-\theta_{1}\right):=1-\kappa \sin \left(\theta_{2}-\theta_{1}\right) .
$$

The scalar dynamics (3) can be analyzed graphically by plotting the vector field $f\left(\theta_{2}-\theta_{1}\right)$ over the difference variable $\theta_{2}-\theta_{1}$, as in Figure 2(a). The non-smoothness of $f\left(\theta_{2}-\theta_{1}\right)$ at the boundaries $\{0, \pi\}$ is an artifact of the nonsmoothness of the geodesic distance on the state space $\mathbb{T}^{2}$.

Figure 2 displays a saddle-node bifurcation at $\kappa=1$ : for $\kappa<1$ no equilibrium of (3) exists, and for $\kappa>1$ an asymptotically stable equilibrium $\left.\theta_{\text {stable }} \in\right] 0, \pi / 2[$ together with a saddle point $\left.\theta_{\text {saddle }} \in\right] \pi / 2, \pi[$ exists. These equilibria satisfy $\sin \left(\theta_{\text {stable }}\right)=\sin \left(\theta_{\text {saddle }}\right)=\kappa^{-1}$. Thus, $\theta_{\text {saddle }}$ and $\theta_{\text {stable }}$ can be shifted arbitrary close to $\pi$, respectively zero, by increasing $\kappa$. For $\theta(0) \in \Delta\left(\left|\theta_{\text {saddle }}\right|\right)$ all trajectories converge (exponentially) to $\theta_{\text {stable }}$, that is, the oscillators synchronize exponentially. Additionally, the oscillators are phase cohesive iff $\theta(0) \in \bar{\Delta}\left(\left|\theta_{\text {saddle }}\right|\right)$, where all trajectories remain bounded. For $\theta(0) \notin \bar{\Delta}\left(\left|\theta_{\text {saddle }}\right|\right)$ the difference $\theta_{2}(t)-\theta_{1}(t)$ will increase beyond $\pi$, and by definition will change its sign since the oscillators change orientation. Ultimately, $\theta_{2}(t)-\theta_{1}(t)$ converges to the equilibrium $\theta_{\text {stable }}$ in the branch where $\theta_{2}-$ $\theta_{1}<0$. In the configuration space $\mathbb{T}^{2}$ this implies that the geodesic distance $\left|\theta_{2}(t)-\theta_{1}(t)\right|$ increases to its maximum

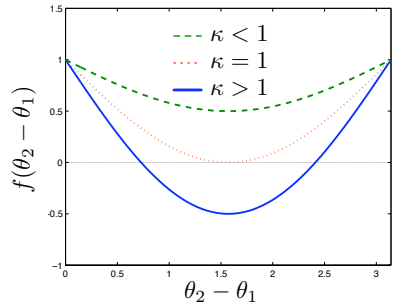

(a) Vector field (3) for $\theta_{2}-\theta_{1}>0$

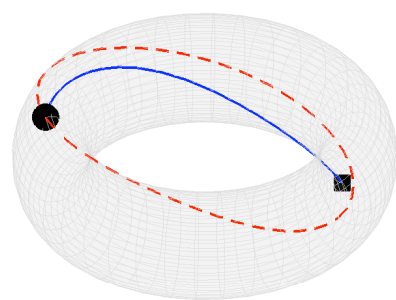

(b) Trajectory $\theta(t)$ for $\kappa=1$
Fig. 2. Plot of the vector field (3) for various values of $\kappa$ and a trajectory $\theta(t) \in \mathbb{T}^{2}$ for the critical case $\kappa=1$, where the dashed line is the equilibrium manifold and $\boldsymbol{\square}$ and $\bullet$ correspond to $\theta(0)$ and $\lim _{t \rightarrow \infty} \theta(t)$. value $\pi$ and shrinks again, that is, the oscillators are not phase cohesive and revolve once around the circle before converging to the equilibrium manifold. Note that for $\kappa \gg 1$ the oscillators practically achieve phase synchronization from every initial condition in an open semi-circle. In the critical case, $\kappa=1$, the saddle point at $\pi / 2$ is globally attractive but not stable: for $\theta_{2}(0)-\theta_{1}(0)=\pi / 2+\epsilon$ (with $\epsilon>0$ sufficiently small), the oscillators are not phase cohesive and revolve around the circle before converging to the saddle equilibrium manifold in $\mathbb{T}^{2}$, as illustrated in the simulation in Figure 2(b). Thus, the saddle equilibrium manifold is both attractor and separatrix which corresponds to a double zero eigenvalue with two dimensional Jordan block in the linearized case. In conclusion, the simple but already rich 2-dimensional case shows that two oscillators are phase cohesive and synchronize if and only if $K>K_{\text {criticial }} \triangleq \omega_{2}-\omega_{1}$, and the ratio $\kappa^{-1}=K_{\text {criticial }} / K<1$ determines the ultimate phase cohesiveness as well as the set of admissible initial conditions. In other words, practical phase synchronization is achieved for $K \gg K_{\text {criticial }}$, and phase cohesiveness occurs only for initial conditions $\left.\theta(0) \in \bar{\Delta}(\gamma), \gamma=\arcsin \left(K_{\text {criticial }} / K\right) \in\right] \pi / 2, \pi[$. This set of admissible initial conditions $\bar{\Delta}(\gamma)$ can be enlarged to an open semi-circle by increasing $K / K_{\text {criticial }}$. Finally, synchronization is lost in a saddle-node bifurcation ${ }^{1}$ at $K=K_{\text {criticial }}$. In Section IV we will generalize all outcomes of this simple analysis to the case of $n$ oscillators.

\section{REVIEW OF BOUNDS FOR THE CRITICAL COUPLing}

In case that all natural frequencies are identical, that is, $\omega_{i} \equiv \omega$ for all $i \in\{1, \ldots, n\}$, a transformation to a rotating frame leads to $\omega \equiv 0$. In this case, the analysis of the Kuramoto model (1) is particularly simple and almost global stability can be derived by various methods. A sample of different analysis schemes includes the contraction property [22], quadratic Lyapunov functions [13], linearization [23], or order parameter and potential function arguments [4].

In the following, we review various analysis methods and the resulting bounds on the critical coupling strength for the case of non-identical frequencies. Most of the following techniques also apply to the case of identical frequencies.

\section{A. Bounds for the Infinite Dimensional Kuramoto Model}

In the physics and dynamical systems community the Kuramoto model (1) is typically studied in the continuum limit as the number of oscillators tends to infinity and the natural frequencies obey an integrable distribution function $g: \mathbb{R} \rightarrow \mathbb{R}_{\geq 0}$. In this case, model (1) is rendered to a first order continuity equation or a second order Fokker-Planck equation when stochasticity is included. Kuramoto himself considered a symmetric, continuous, and unimodal distribution $g(\omega)$ centered above zero and showed in an insightful and ingenuous analysis [1], [8] that the incoherent state (i.e., a uniform distribution of the oscillators on the unit circle) supercritically bifurcates for the critical coupling strength

$$
K_{\text {critical }}=\frac{2}{\pi g(0)} .
$$

${ }^{1}$ For Kuramoto oscillator models of dimension $n \geq 3$, this loss of synchrony via a saddle-node bifurcation at $K_{\text {criticial }}$ is only the starting point of a series of bifurcation occurring if $K$ is further decreased, see [26]. 
The bound (4) for the on-set of synchronization has also been derived in [2], [3]. In [9] Ermentrout considered symmetric distributions $g(\omega)$ with bounded domain $\omega \in\left[-\omega_{\max }, \omega_{\max }\right]$, and studied the existence of phase-locked solutions. The coupling threshold $K_{\text {critical }}$ necessary for the existence of phaselocked solution reads in our notation as [9, Proposition 2]

$$
\frac{\omega_{\max }}{K_{\text {critical }}}=\max _{p \in \mathbb{R}, p \geq 1}\left\{\frac{1}{p^{2}} \int_{-1}^{1} \sqrt{p^{2}-\omega^{2}} g(\omega) d \omega\right\} .
$$

Ermentrout further showed that formula (5) yields $K_{\text {critical }} \geq$ $2 \omega_{\max }$ for symmetric distributions and $K_{\text {critical }} \geq 4 \omega_{\max } / \pi$ whenever $g$ is non-increasing in $[0,1]$. Both of these bounds are tight for a bipolar (i.e., a bimodal double-delta) distribution and a uniform distribution [9, Corollary 2], [11, Sections 3 \& 4]. Similar results for the bipolar distribution are also obtained in [3], and a bimodal Lorentzian distribution is analyzed in [10]. For various other references analyzing the continuum limit of the Kuramoto model we refer to [2], [3].

\section{B. Explicit and Necessary or Sufficient Bounds for the Finite Dimensional Kuramoto Model}

For the finite dimensional Kuramoto model (1), we assume that the natural frequencies are supported on a compact interval $\omega_{i} \in\left[\omega_{\max }, \omega_{\min }\right] \subset \mathbb{R}, i \in\{1, \ldots, n\}$. This assumption can be made without loss of generality since the critical coupling $K_{\text {critical }}$ is not finite for unbounded natural frequencies $\omega_{i}$ [19, Theorem 1]. In [12], [13] a necessary condition for the existence of synchronized solutions is given in terms of the width of the interval $\left[\omega_{\max }, \omega_{\min }\right]$ as

$$
K>\frac{n\left(\omega_{\max }-\omega_{\min }\right)}{2(n-1)} .
$$

Obviously, in the limit as $n \rightarrow \infty$, this bound reduces $\left(\omega_{\max }-\right.$ $\left.\omega_{\min }\right) / 2$, the simple bound derived in the introduction of this paper. A looser but still insightful necessary condition is $K \geq$ $2 \sigma$, where $\sigma$ is the variance of the $\omega_{i}$ [11], [19, Corollary $2]$. For bipolar distributions $\omega_{i} \in\left\{\omega_{\min }, \omega_{\max }\right\}$, necessary explicit conditions similar to (6) can be derived for noncomplete and highly symmetric coupling topologies [25].

Various bounds sufficient for synchronization have been derived including estimates of the region of attraction [7], [12]-[18]. Typically, these sufficient bounds are based on incremental stability arguments and are of the form

$$
K>\|V \omega\|_{p} \cdot f(n, \gamma),
$$

where $\|\cdot\|_{p}$ is the $p$-norm and $V$ is a matrix (of yet unspecified row dimension) measuring the non-uniformity among the $\omega_{i}$. For instance, $V=I_{n}-(1 / n) \mathbf{1}_{n \times n}$ gives the $i$ th component of $V \omega$ as $\omega_{i}-\omega_{\text {avg }}$, that is, as deviation from the average natural frequency. Finally, the function $f: \mathbb{N} \times\left[0, \pi / 2\left[\rightarrow\left[1, \infty\left[\right.\right.\right.\right.$ captures the dependence of $K_{\text {critical }}$ on the number of oscillators $n$ and the scalar $\gamma$ determining a bound on the admissible pairwise phase differences, which is, for instance, of the form $\left\|\left(\ldots, \theta_{i}(t)-\theta_{j}(t), \ldots\right)\right\|_{p} \leq \gamma$.

Two-norm bounds, i.e., $p=2$ in condition (7), have been derived using quadratic Lyapunov functions in [12, proof of Theorem 4.2] and [7, Theorem V.9], where the matrix $V \in \mathbb{R}^{n(n-1) / 2 \times n}$ is the incidence matrix such that $V \omega$ is the vector of $n(n-1) / 2$ pairwise differences $\omega_{i}-\omega_{j}$. A sinusoidal Lyapunov function [15, Proposition 1] leads to a two-norm bound with $V=I_{n}-(1 / n) \mathbf{1}_{n \times n}$. Similar twonorm bounds have been obtained by contraction mapping [13, Theorem 2] and by contraction analysis [14, Theorem 3.8], where $V \in \mathbb{R}^{n-1 \times n}$ is an orthonormal projector on the subspace orthogonal to $\mathbf{1}_{n \times 1}$. For all cited references the region of attraction is given by the initial phase differences in two-norm or $\infty$-norm balls satisfying $\|V \theta(0)\|_{2, \infty}<\pi$. Unfortunately, none of these bounds scales independently of $n$ since $\|V \omega\|_{2}^{2}$ is a sum of at least $n-1$ terms in all cited references and $f(n, \gamma)$ in condition (7) is either an increasing [13] or a constant function of $n$ [7], [12], [14], [15].

A scaling of condition (7) independently of $n$ has been achieved only when considering the width $\omega_{\max }-\omega_{\min }=$ $\left\|\left(\ldots, \omega_{i}-\omega_{j}, \ldots\right)\right\|_{\infty}$, that is, for $V \omega$ being the vector of all $n(n-1) / 2$ pairwise frequency differences and $p=\infty$ in condition (7). A quadratic Lyapunov function leads to $f(n, \gamma)=n /(2 \sin (\gamma))$ [12, proof of Theorem 4.1], a contraction argument leads to $f(n, \gamma)=n /((n-2) \sin (\gamma))$ [16, Lemma 9], and a geometric argument leads to the scale-free bound $f(\gamma)=1 /(2 \sin (\gamma / 2) \cos (\gamma))$ [17, proof of Proposition 1]. In [18, Theorem 3.3] and in our earlier work [7, Theorem V.3], the simple and scale-free bound $f(\gamma)=1 / \sin (\gamma)$ has been derived by analyticity and contraction arguments. In our notation, the region of attraction for synchronization is in all the cited references [7], [12], [16]-[18] given as $\theta(0) \in \bar{\Delta}(\gamma)$ for $\gamma \in[0, \pi / 2[$.

\section{Implicit and Exact Bounds for the Finite Dimensional Kuramoto Model}

Three recent articles [19]-[21] independently derived a set of implicit consistency equations for the exact critical coupling strength $K_{\text {critical }}$ for which phase-locked solutions exist. Verwoerd and Mason provided the following implicit formula to compute $K_{\text {critical }}$ [19, Theorem 3]:

$$
K_{\text {critical }}=n u^{*} / \sum_{i=1}^{n} \sqrt{1-\left(\Omega_{i} / u^{*}\right)^{2}}
$$

where $\Omega_{i}=\omega_{i}-\frac{1}{n} \sum_{j=1}^{n} \omega_{j}$ and $u^{*} \in\left[\|\Omega\|_{\infty}, 2\|\Omega\|_{\infty}\right]$ is the unique solution to the implicit equation

$$
2 \sum_{i=1}^{n} \sqrt{1-\left(\Omega_{i} / u^{*}\right)^{2}}=\sum_{i=1}^{n} 1 / \sqrt{1-\left(\Omega_{i} / u^{*}\right)^{2}} .
$$

Verwoerd and Mason also extended their results to bipartite graphs [24] but did not carry out a stability analysis. The formulas (8)-(9) can be reduced exactly to the implicit selfconsistency equation derived by Mirollo and Strogatz in [20] and by Aeyels and Rogge in [21], where additionally a local stability analysis is carried out. The stability analysis [20], [21] in the $n$-dimensional case shows the same sadlenode bifurcation as the two-dimensional Example II.1: for $K<K_{\text {critical }}$ there exist no phase-locked solutions $\theta(t)$, for $K>K_{\text {critical }}$ there exist stable phase-locked solutions $\theta(t)$, and for $K=K_{\text {critical }}$ the Jacobians of phase-locked solutions equilibria have a double zero eigenvalue with twodimensional Jordan block, as illustrated in Example II.1.

In conclusion, in the finite dimensional case various necessary or sufficient explicit bounds on the coupling strength 
$K_{\text {critical }}$ are known as well as implicit formulas to compute $K_{\text {critical }}$ which is provably a threshold for local stability.

IV. An EXPLICIT, NECESSARY, AND SUFFICIENT

\section{Condition on the CRitical Coupling Strength}

From the point of analyzing or designing a sufficiently strong coupling in the Kuramoto-type applications [2]-[7], the exact bound (8)-(9) has three drawbacks. First, it is implicit and thus not suited for performance or robustness estimates in case of additional coupling strength, e.g., which level of ultimate phase cohesiveness or which magnitude of the order parameter can be achieved for $K=c \cdot K_{\text {critical }}$ with a certain $c>1$. Second, the corresponding region of attraction of a phase-locked equilibrium for a given $K>$ $K_{\text {critical }}$ is unknown. Finally, the particular natural frequencies $\omega_{i}$ (or their distributions) are typically time-varying, uncertain, or even unknown in most biological or technological applications [2]-[7]. In this case, the exact $K_{\text {critical }}$ has to be dynamically estimated and re-computed over time, or a conservatively strong coupling $K \gg K_{\text {critical }}$ has to be chosen. Unfortunately, none of the explicit sufficient bounds (7) appears to be tight to the exact implicit bound (8)-(9).

The following theorem states an explicit bound on the coupling strength together with performance estimates, convergence rates, and a guaranteed semi-global region of attraction for synchronization. Besides improving all other bounds known to the authors, our bound is tight and thus necessary and sufficient when considering arbitrary distributions of the natural frequencies supported on a compact interval.

Theorem IV.1 (Explicit, necessary, and sufficient synchronization condition) Consider the Kuramoto model (1) with natural frequencies $\left(\omega_{1}, \ldots, \omega_{n}\right)$ and coupling strength $K$. The following three statements are equivalent:

(i) the coupling strength $K$ is larger than the maximum non-uniformity among the natural frequencies, i.e.,

$$
K>K_{\text {critical }} \triangleq \omega_{\max }-\omega_{\min }
$$

(ii) there exists an arc length $\left.\left.\gamma_{\max } \in\right] \pi / 2, \pi\right]$ such that the Kuramoto model (1) synchronizes exponentially for all possible distributions of the natural frequencies supported on $\left[\omega_{\min }, \omega_{\max }\right]$ and for all initial phases $\theta(0) \in \Delta\left(\gamma_{\max }\right)$; and

(iii) there exists a locally exponentially stable synchronized trajectory $\theta: \mathbb{R}_{\geq 0} \rightarrow \mathbb{T}^{n}$ in $\bar{\Delta}\left(\gamma_{\min }\right)$ for some arc length $\gamma_{\min } \in[0, \pi / 2[$ and for all possible distributions of the natural frequencies supported on $\left[\omega_{\min }, \omega_{\max }\right]$.

If the three equivalent cases (i), (ii), and (iii) hold, then the ratio $K_{\text {critical }} / K$ and the arc lengths $\gamma_{\min } \in[0, \pi / 2[$ and $\left.\left.\gamma_{\max } \in\right] \pi / 2, \pi\right]$ are related uniquely via $\sin \left(\gamma_{\min }\right)=$ $\sin \left(\gamma_{\max }\right)=K_{\text {critical }} / K$, and the following statements hold:

1) phase cohesiveness: the set $\bar{\Delta}(\gamma)$ is positively invariant for every $\gamma \in\left[\gamma_{\min }, \gamma_{\max }\right]$, and each trajectory starting in $\Delta\left(\gamma_{\max }\right)$ approaches asymptotically $\bar{\Delta}\left(\gamma_{\min }\right)$;

2) order parameter: the asymptotic value of the magnitude $r_{\infty}$ of the order parameter is bounded as

$$
1 \geq r_{\infty} \geq \cos \left(\frac{\gamma_{\min }}{2}\right)=\sqrt{\frac{1+\sqrt{1-\left(K_{\text {critical }} / K\right)^{2}}}{2}} ;
$$

3) frequency synchronization: the synchronization frequency is the average frequency $\omega_{\mathrm{avg}}=\frac{1}{n} \sum_{i=1}^{n} \omega_{i}$, and, given phase cohesiveness in $\bar{\Delta}(\gamma), \gamma \in[0, \pi / 2[$, the exponential synchronization rate is no worse than $\lambda_{\mathrm{fs}}=-K \cos (\gamma) ;$ and

4) phase synchronization: if $\omega_{i}=\omega \in \mathbb{R}$ for all $i \in$ $\{1, \ldots, n\}$, then for every $\theta(0) \in \bar{\Delta}(\gamma), \gamma \in[0, \pi[$, the phases synchronize exponentially to the average phase $\theta_{\text {avg }}(t):=\frac{1}{n} \sum_{i=1}^{n} \theta(0)+\omega t$ and the exponential synchronization rate is no worse than $\lambda_{\mathrm{ps}}=-K \operatorname{sinc}(\gamma)$.

To compare the bound (10) to the bounds presented in Section III, we note from the proof of Theorem IV.1 that our bound (10) can be equivalently stated as $K>\left(\omega_{\max }-\right.$ $\left.\omega_{\min }\right) / \sin (\gamma)$ and thus improves the sufficient bounds [12][17]. In the simple case $n=2$ analyzed in Example II.1, the bound (10) is obviously exact and also equals the necessary bound (6). Furthermore, Theorem IV.1 fully generalizes the observations in Example II. 1 to the $n$-dimensional case. In the infinite-dimensional case the bound (10) is tight with respect to the necessary bound for a bipolar distribution $\omega_{i} \in$ $\left\{\omega_{\min }, \omega_{\max }\right\}$ derived in [3], [9], [11]. Note that condition (10) guarantees synchronization for arbitrary distributions of $\omega_{i}$ supported in $\left[\omega_{\min }, \omega_{\max }\right]$, which can possibly be uncertain, time-varying (addressed in detail in [27]), or even unknown. Theorem IV.1 also guarantees a larger region of attraction $\theta(0) \in \Delta\left(\gamma_{\max }\right)$ for synchronization than [7], [12]-[18].

Besides the necessary and sufficient bound (10), Theorem IV.1 gives guaranteed exponential convergence rates for frequency and phase synchronization, and it establishes a practical stability result in the sense that the multiplicative gap $K_{\text {critical }} / K$ in the bound (10) determines the admissible initial and the guaranteed ultimate phase cohesiveness as well as the guaranteed asymptotic magnitude $r$ of the order parameter. In view of this result, the convergence properties of the Kuramoto model (1) are best described by the controltheoretical terminology "practical phase synchronization."

The proof of Theorem IV.1 is detailed in [27] and is only briefly sketched here. Similar to [7], [16]-[18], the proof of sufficiency (i) $\Rightarrow$ (ii) relies on a contraction argument in combination with a consensus analysis: if condition (10) holds, then the Lyapunov function $V(\theta(t))=$ $\max _{i, j \in\{1, \ldots, n\}}\left|\theta_{i}(t)-\theta_{j}(t)\right|$ guarantees the phase cohesiveness and phase synchronization, as stated in 1) and 4). Statement 2) on the order parameter follows then immediately from Lemma II.1. Notice that the frequencies $\dot{\theta}_{i}(t)$ of the Kuramoto model obey the linear time-varying and symmetric consensus dynamics $\frac{d}{d t} \dot{\theta}_{i}=\sum_{j=1}^{n} a_{i j}(t)\left(\dot{\theta}_{j}-\right.$ $\left.\dot{\theta}_{i}\right)$, where all terms $a_{i j}(t)=a_{j i}(t)=(K / n) \cos \left(\theta_{i}(t)-\theta_{j}(t)\right)$ become strictly positive in finite time due to 1$)$. Thus, a standard consensus analysis yields the frequency synchronization stated in 3). In summary, condition (10) implies (ii) and 1)4). The necessity (ii) $\Rightarrow$ (i) is proven by showing that the bound (10) is tight: if (i) is not satisfied, then exponential synchronization cannot occur for a bipolar distribution of the natural frequencies $\omega_{i} \in\left\{\omega_{\min }, \omega_{\max }\right\}$. Finally, the equivalence (i), (ii) $\Leftrightarrow$ (iii) follows from the definition of exponential synchronization and by basic arguments from ordinary differential equations, similar to [7, Lemma IV.1]. 


\section{Simulation Studies}

Theorem IV.1 places a hard bound on the critical coupling strength $K_{\text {critical }}$ for all distributions of $\omega_{i}$ supported on the compact interval $\left[\omega_{\max }, \omega_{\min }\right]$, including the worst-case bipolar distribution. For a particular distribution $g(\omega)$ supported on $\left[\omega_{\min }, \omega_{\max }\right]$ the bound (10) is only sufficient and possibly a factor 2 larger than the necessary bound (6). The exact critical coupling for $g(\omega)$ lies somewhere in between and can be obtained by solving the implicit equations (8)-(9).

The following example illustrates the average case for natural frequencies sampled from a uniform distribution $g(\omega)=1 / 2$ supported for $\omega \in[-1,1]$. Figure 3 reports numerical findings on the critical coupling strength for $n \in$ $[2,300]$ oscillators in a semi-log plot, where the coupling strengths for each $n$ are averaged over 1000 simulations.

First, note that the three displayed bounds are equivalent for $n=2$ oscillators. As the number of oscillators increases, the sufficient bound (10) clearly converges to $\omega_{\max }-\omega_{\min }=$ 2 , the width of the distribution $g(\omega)$, and the necessary bound (6) accordingly to half of the width. The exact bound (8)-(9) quickly converges to $4\left(\omega_{\max }-\omega_{\min }\right) /(2 \pi)=4 / \pi$ in agreement with the results (4) and (5) predicted for the continuum limit case. It can be observed that the exact bound (8)-(9) is closer to the tight bound (10) for a small number of oscillators, i.e., when there are few outliers increasing the width $\omega_{\max }-\omega_{\min }$. For large $n$, the sample size of $\omega_{i}$ increases and thus also the number of outliers. In this case, the exact bound (8)-(9) is closer to the necessary bound (6).

\section{CONCLUSIONS}

This paper reviewed various bounds on the critical coupling strength in the Kuramoto model, formally introduced the powerful concept of phase cohesiveness, and presented an explicit and tight bound sufficient for synchronization in the finite-dimensional Kuramoto model. This bound is necessary and sufficient for arbitrary distributions of the natural frequencies and tight for the particular case, where only implicit bounds are known. Furthermore, a general practical stability result as well as various performance measures have been derived as a function of the multiplicative gap in the bound.

In view of the different biological and technological applications of the Kuramoto model [2]-[7], similar tight and explicit bounds have to be derived for synchronization (as well as splay state stabilization) with arbitrary coupling topologies, possibly non-uniform and time-varying coupling weights, phase and time delays, and higher order dynamics.

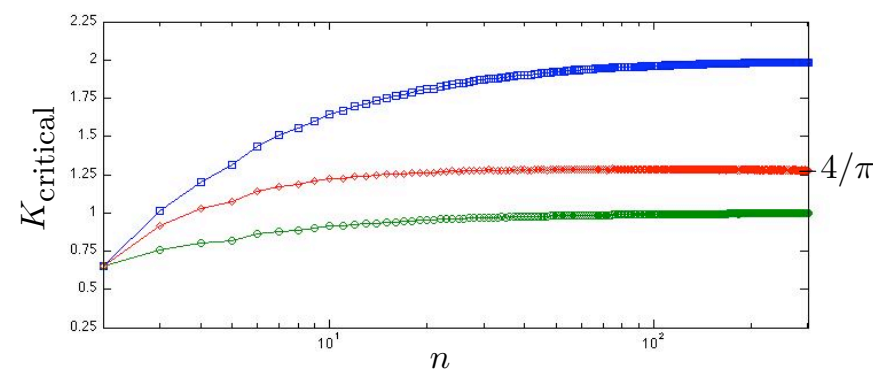

Fig. 3. Comparison of the necessary bound (6) (O), the exact and implicit bound $(8)-(9)(\diamond)$, and the tight and explicit bound $(10)(\square)$ on $K_{\text {critical }}$.

\section{REFERENCES}

[1] Y. Kuramoto, "Self-entrainment of a population of coupled nonlinear oscillators," in Int. Symposium on Mathematical Problems in Theoretical Physics, ser. Lecture Notes in Physics, H. Araki, Ed. Springer Verlag, 1975, vol. 39, pp. 420-422.

[2] S. H. Strogatz, "From Kuramoto to Crawford: Exploring the onset of synchronization in populations of coupled oscillators," Physica D, vol. 143, no. 1, pp. 1-20, 2000

[3] J. A. Acebron, L. L. Bonilla, C. J. P. Vicente, F. Ritort, and R. Spigler, "The Kuramoto model: A simple paradigm for synchronization phenomena," Reviews of Modern Physics, vol. 77, no. 1, pp. 137-185, 2005

[4] R. Sepulchre, D. A. Paley, and N. E. Leonard, "Stabilization of planar collective motion: All-to-all communication," IEEE Trans Automatic Ctrl, vol. 52, no. 5, pp. 811-824, 2007.

[5] K. Wiesenfeld, P. Colet, and S. H. Strogatz, "Frequency locking in Josephson arrays: Connection with the Kuramoto model," Physical Review E, vol. 57, no. 2, pp. 1563-1569, 1998.

[6] P. A. Tass, "A model of desynchronizing deep brain stimulation with a demand-controlled coordinated reset of neural subpopulations," Biological Cybernetics, vol. 89, no. 2, pp. 81-88, 2003.

[7] F. Dörfler and F. Bullo, "Synchronization and transient stability in power networks and non-uniform Kuramoto oscillators," Oct. 2009 available at http://arxiv.org/abs/0910.5673.

[8] Y. Kuramoto, Chemical Oscillations, Waves, and Turbulence. Springer Verlag, 1984

[9] G. B. Ermentrout, "Synchronization in a pool of mutually coupled oscillators with random frequencies," Journal of Mathematical Biology, vol. 22 , no. 1 , pp. $1-9,1985$.

[10] E. A. Martens, E. Barreto, S. H. Strogatz, E. Ott, P. So, and T. M. Antonsen, "Exact results for the Kuramoto model with a bimodal frequency distribution," Physical Review E, vol. 79, no. 2, p. 26204 , 2009

[11] J. L. van Hemmen and W. F. Wreszinski, "Lyapunov function for the Kuramoto model of nonlinearly coupled oscillators," Journal of Statistical Physics, vol. 72, no. 1, pp. 145-166, 1993

[12] N. Chopra and M. W. Spong, "On exponential synchronization of Kuramoto oscillators," IEEE Trans Automatic Ctrl, vol. 54, no. 2, pp. 353-357, 2009.

[13] A. Jadbabaie, N. Motee, and M. Barahona, "On the stability of the Kuramoto model of coupled nonlinear oscillators," in Proc ACC, Boston, MA, June 2004, pp. 4296-4301.

[14] S. Chung and J. Slotine, "On synchronization of coupled HopfKuramoto oscillators with phase delays," Apr. 2010, available at http://arxiv.org/abs/1004.5366.

[15] A. Franci, A. Chaillet, and W. Pasillas-Lépine, "Phase-locking between Kuramoto oscillators: Robustness to time-varying natural frequencies,' in Proc CDC, 2010, submitted.

[16] G. S. Schmidt, U. Münz, and F. Allgöwer, "Multi-agent speed consensus via delayed position feedback with application to Kuramoto oscillators," in Proc ECC, Budapest, Hungary, Aug. 2009, pp. 2464 2469.

[17] F. De Smet and D. Aeyels, "Partial entrainment in the finite KuramotoSakaguchi model," Physica D, vol. 234, no. 2, pp. 81-89, 2007.

[18] S.-Y. Ha, T. Ha, and J.-H. Kim, "On the complete synchronization of the Kuramoto phase model," Physica D, vol. 239, no. 17, pp. 1692 $1700,2010$.

[19] M. Verwoerd and O. Mason, "Global phase-locking in finite populations of phase-coupled oscillators," SIAM Journal on Applied Dynamical Systems, vol. 7, no. 1, pp. 134-160, 2008.

[20] R. E. Mirollo and S. H. Strogatz, "The spectrum of the locked state for the Kuramoto model of coupled oscillators," Physica D, vol. 205, no. 1-4, pp. 249-266, 2005.

[21] D. Aeyels and J. A. Rogge, "Existence of partial entrainment and stability of phase locking behavior of coupled oscillators," Progress on Theoretical Physics, vol. 112, no. 6, pp. 921-942, 2004.

[22] Z. Lin, B. Francis, and M. Maggiore, "State agreement for continuoustime coupled nonlinear systems," SIAM JCO, vol. 46, no. 1, pp. 288 307, 2007.

[23] E. Canale and P. Monzón, "Almost global synchronization of symmetric Kuramoto coupled oscillators," in Systems Structure and Control. InTech Education and Publishing, 2008, ch. 8, pp. 167-190.

[24] M. Verwoerd and O. Mason, "On computing the critical coupling coefficient for the kuramoto model on a complete bipartite graph," SIAM Journal on Applied Dynamical Systems, vol. 8, no. 1, pp. 417453, 2009.

[25] L. Buzna, S. Lozano, and A. Diaz-Guilera, "Synchronization in symmetric bipolar population networks," Physical Review E, vol. 80, no. 6, p. 66120, 2009.

[26] Y. Maistrenko, O. Popovych, and P. Tass, "Desynchronization and chaos in the Kuramoto model," in Dynamics of Coupled Map Lattices and of Related Spatially Extended Systems, ser. Lecture Notes in Physics, J.-R. Chazottes and B. Fernandez, Eds. Springer Verlag, 2005, vol. 671, pp. 285-306.

[27] F. Dörfler and F. Bullo, "On the critical coupling for Kuramoto oscillators," SIAM J Applied Dynamical Systems, Nov. 2010, submitted. 